\title{
Effectiveness and Tolerability of Oral Amoxicillin in Pregnant Women with Active Syphilis, Japan, 2010-2018
}

\author{
Takeshi Nishijima, Kei Kawana, Ichio Fukasawa, Naoko Ishikawa, \\ Melanie M. Taylor, Hiroshige Mikamo, Kiyoko Kato, Jo Kitawaki, Tomoyuki Fujii, \\ Women's Health Care Committee, Japan Society of Obstetrics and Gynecology
}

We conducted a nationwide retrospective study in Japan to evaluate the effectiveness of oral amoxicillin or ampicillin as alternatives to injectable benzathine penicillin $G$ for treating pregnant women with syphilis and preventing congenital syphilis (CS). We investigated 80 pregnant women with active syphilis treated with amoxicillin or ampicillin during 2010-2018. Overall, 21\% (15/71) had pregnancies resulting in CS cases, and $3.8 \%(3 / 80)$ changed therapies because of side effects. Among 26 patients with early syphilis, no CS cases occurred, but among 45 with late syphilis, 15 (33\%) CS cases occurred. Among 57 patients who started treatment $\geq 60$ days before delivery, $8(14 \%)$ had CS pregnancy outcomes. We found oral amoxicillin potentially ineffective for preventing CS cases among pregnant women with late syphilis but potentially effective in those with early syphilis. Prospective studies are needed to definitively evaluate the efficacy of amoxicillin for the treatment of pregnant women with syphilis to prevent CS.

Cyphilis is a sexually transmitted infection that can $\checkmark$ be passed from mother to infant during pregnancy and childbirth. Mother-to-child transmission (MTCT)

Author affiliations: National Center for Global Health and Medicine, Tokyo, Japan (T. Nishijima); World Health Organization Regional Office of the Western Pacific, Manila, the Philippines (T. Nishijima, N. Ishikawa); Nihon University School of Medicine, Tokyo, Japan (K. Kawana); Dokkyo Medical University, Tochigi, Japan (I. Fukasawa); World Health Organization, Geneva, Switzerland (M.M. Taylor); Centers for Disease Control and Prevention, Atlanta, Georgia, USA (M.M. Taylor); Aichi Medical University, Aichi, Japan (H. Mikamo); Kyushu University Graduate School of Medical Sciences, Fukuoka, Japan (K. Kato); Graduate School of Medical Science, Kyoto Prefectural University of Medicine, Kyoto, Japan (J. Kitawaki); Graduate School of Medicine, The University of Tokyo, Tokyo, Japan (T. Fujii)

DOI: https://doi.org/10.3201/eid2606.191300 of syphilis results in congenital syphilis (CS), which can cause serious outcomes, including miscarriage, stillbirth, neonatal death, preterm birth, low birth weight, and various illnesses and congenital deformities. The World Health Organization (WHO) estimated 988,000 active syphilis cases and 611,000 CS cases in pregnant women worldwide in 2016 (1), and syphilis is the second most common infectious cause of stillbirth worldwide (2).

Injectable benzathine penicillin G (BPG) is the only regimen recommended in WHO and US Centers for Disease Control and Prevention (CDC) guidelines (3-5) for the treatment of syphilis in pregnant women to prevent CS. Sufficient evidence is not available to recommend an alternative regimen. In a systematic review on alternative treatments for pregnant women with syphilis, only 21 pregnant women treated with regimens other than BPG could be identified (6). Erythromycin and azithromycin do not cross the placental barrier and thus cannot treat infections in the fetus (6). Macrolide-resistant T. pallidum has been reported in many countries (7). Tetracycline and doxycycline are contraindicated in the second and third trimesters of pregnancy, and data are not sufficient to recommend ceftriaxone for treatment of maternal syphilis and prevention of CS $(4,8)$. During 2014-2016, shortages or stockouts of BPG were reported by 39 of 95 surveyed countries and territories (9), and in several countries, including Japan, intramuscular BPG is not available (10). A reliable alternative treatment for syphilis in pregnant women to prevent CS is urgently needed.

Because sales of BPG stopped in Japan in 1986, oral penicillins, such as amoxicillin or ampicillin, have been primarily used to treat pregnant women with syphilis. These regimens are indicated in the obstetrics and gynecology care guidelines of Japan 
(11), which follows the regimens recommended by the Japanese Society for Sexually Transmitted Infections diagnosis and treatment guidelines (12). These guidelines recommend oral amoxicillin or ampicillin with dosing of $1,500 \mathrm{mg} /$ day (i.e., $500 \mathrm{mg} \mathrm{3 \times /d}$ ) for 2-4 weeks for primary syphilis, 4-8 weeks for secondary syphilis, and 8-12 weeks for tertiary or later-stage syphilis in pregnant women. However, effectiveness of this regimen for pregnant women and prevention of CS has not been reported, except for 1 pregnant woman who was treated with a much higher dosage of amoxicillin $(6 \mathrm{~g} / \mathrm{d})$ plus probenecid $(1 \mathrm{~g} / \mathrm{d})$ for 14 days (13). In this retrospective study, we investigated the effectiveness and tolerability of oral amoxicillin and ampicillin in pregnant women to treat active syphilis and prevent MTCT of syphilis.

\section{Methods}

\section{Study Setting and Population}

We conducted this nationwide, multicenter retrospective study in Japan as a joint research project between the Japan Society of Obstetrics and Gynecology and the WHO (Appendix, https://wwwnc.cdc.gov/ EID/article/26/6/19-1300-App1.pdf) (14). The study was approved by the human research ethics committees of Nihon University School of Medicine (Tokyo, Japan), Dokkyo Medical University (Tochigi, Japan), and the Japan Society of Obstetrics and Gynecology (Tokyo, Japan). We also submitted the study protocol to the WHO Ethics Review Committee. Because our research involved anonymized patient data and not new data collection or an additional intervention, the study was determined to be exempt from WHO Ethics Review Committee review. The requirement for informed consent was waived because this study included only data gained from routine clinical practice. We conducted this study according to the principles expressed in the Declaration of Helsinki.

We included pregnant women with syphilis, regardless of their symptoms, who were treated with oral amoxicillin or ampicillin during 2010-2018; we only included those with serum rapid plasma reagin (RPR) titers $\geq 8$ and positive results from treponemal tests, such as the T. pallidum hemagglutination test $(10,15)$. We excluded patients with tertiary syphilis or neurosyphilis diagnoses that were based on findings from cerebrospinal fluid (CSF) samples $(10,16)$ or patient symptoms (including ocular or auditory syphilis) and those with suspected reinfections after initiation of syphilis treatment (defined as patients with $\geq 4$-fold rise in RPR titer after 4-fold decrement with or without symptoms) $(10,15,17)$.
In Japan, the automated latex turbidimetric immunoassay and the conventional manual RPR card test are both used to determine RPR titers $(18,19)$. The automated RPR test highly correlates with the manual test for syphilis diagnoses $(18,19)$; just like the manual card test, for the automated test, a 4-fold decrement in RPR titer is a good criterion for successful treatment of syphilis (19). Thus, we treated the titers from the automated RPR test the same as those from the manual RPR test.

\section{Definitions}

We defined CS following the reporting criteria set by the Japanese Ministry of Health, Labour, and Welfare (20). Diagnosis of CS in newborns required fulfillment of any 1 of the following criteria: serum RPR titer $\geq 4$-fold that of the mother's (4); fluorescent treponemal antibody absorption (FTA-ABS) test result positive for serum $\operatorname{IgM}(12,21,22)$; lesion tissue or body fluid samples positive by PCR (4); lesion or fluid samples positive for T. pallidum on dark field microscopy (4); or physical examination findings consistent with CS, such as nonimmune hydrops, jaundice, hepatosplenomegaly, skin rash, pseudoparalysis, and rhinitis $(4,20,23)$. We classified the mother's stage of syphilis into early syphilis (primary, secondary, or early latent syphilis) and late syphilis (late latent syphilis or latent syphilis of unknown duration). We defined early latent syphilis as asymptomatic syphilis that could be linked to reported syphilis symptoms, a sexual exposure, or conversion from a prior negative syphilis test $\leq 1$ year after diagnosis. These criteria occurring $>1$ year after diagnosis constituted a late latent case and at an undefined time point after diagnosis as a latent case of unknown duration $(4,10,24)$. We defined miscarriage as the loss of a fetus before week 20 of pregnancy and stillbirth as the loss during or after week 20.

\section{Data Collection, Outcome Measures, and Statistical Analysis}

We collected data from medical charts at each facility, using a case report form (Appendix). The primary outcome of this study was effectiveness of prevention of MTCT of syphilis. We defined MTCT as a CS case and defined CS cases as live newborns with CS diagnoses, miscarriages, or still births. If a live newborn was not given a CS diagnosis, we interpreted the treatment given as successful for preventing MTCT (5). We evaluated the secondary outcome serologic effectiveness of treatment in the mother (4-fold decrement of serum RPR titer) at each of the following time points: delivery (25), 6 months after therapy (4), and 12 months after 
therapy (4). We analyzed the primary outcome among all study patients and among those who initiated treatment $\geq 60$ days before delivery. We used this subgroup to evaluate drug effectiveness among patients who initiated treatment within an adequate interval (defined by $\mathrm{WHO}$ as $\geq 30$ days before delivery) (26). We used 60 days for this evaluation because of the long treatment duration recommended for oral penicillins in Japan's guidelines (12). We compared the percentage of CS cases between those who had early syphilis and late syphilis, between those treated with amoxicillin and those treated with ampicillin, and between those who were and were not Japanese.

We compared characteristics between groups using the Student $t$-test for continuous variables and using either the $X^{2}$ test or Fisher exact test for categorical variables. We defined statistical significance as a 2 -sided $p$ value $<0.05$. We developed a multivariate logistic regression model to determine the effect of factors on CS cases. We performed this analysis with SAS version 9.4 (https://www.sas.com) and all other statistical analyses with SPSS Statistics 23 (https://www.ibm.com).

\section{Results}

Of 88 hospitals invited into the study, 44 (50\%) participated and provided 131 case report forms (Appendix Figure). We excluded 51 cases, resulting in 80 cases being included in our analysis.

Median patient age was 23 (interquartile range [IQR] 21-27) years; 75 (94\%) patients were Japanese, and only 1 patient was HIV positive (Table 1 ). In total, 31 patients had early syphilis (5 primary, 19 secondary, 7 early latent) and 49 had late syphilis (4 latent, 45 latent with unknown duration). Median RPR titer at diagnosis was 51 (IQR 29-72), and 66 (83\%) patients were treated with amoxicillin and $14(17 \%)$ with ampicillin. The median duration of treatment for pregnant women was 60 (IQR 29-90) days, and the median duration of treatment before delivery was 56 (28-86) days. In total, $61(76 \%)$ patients were treated with $1,500 \mathrm{mg} /$ day dosing, and 3 were concurrently prescribed probenecid. Median gestational age of fetus at CS diagnosis was 13.8 (IQR 11.7-26.3) weeks and at treatment initiation 15.8 (13.0-27.1) weeks. In total, 57 $(71 \%)$ patients received syphilis diagnoses when the fetus had the gestational age of $<20$ weeks. A comparison of pregnant women by syphilis stage revealed that women with late syphilis and early syphilis had similar characteristics but women with late syphilis had longer durations of treatment (Table 2).

Of the 80 cases, we excluded 9 (6 with unknown outcomes, 3 involving abortions induced at 15-17 weeks of pregnancy) from the outcome analysis. Of the remaining 71 cases, 15 (21\%, 95\% CI 13.2\%-32\%) were classified as CS (13 live newborns with CS diagnoses, 1 miscarriage, 1 stillbirth; Tables 2, 3; Appendix Table 1). Effectiveness of treatment for preventing CS cases was significantly better among patients with early syphilis than late syphilis; CS cases developed in $0 \%(0 / 26,95 \%$ CI $0 \%-12.9 \%)$ of patients with early syphilis and $33 \%(15 / 45,95 \%$ CI $21.4 \%-47.9 \%$; $\mathrm{p}<0.001)$ of patients with late syphilis.

Among pregnant women with early syphilis, 26 $(84 \%)$ received $1,500 \mathrm{mg} /$ day of either amoxicillin or ampicillin, and the median duration of antimicrobial drug treatment was 30 (IQR 28-64) days (Table 2). Among those with late syphilis, 35 (71\%) received $1,500 \mathrm{mg} /$ day of either amoxicillin or ampicillin, and the median duration of antimicrobial drug treatment was 78 (IQR 51-104) days. CS cases developed in 19\% $(11 / 58,95 \%$ CI $10.9 \%-30.9 \%)$ of those treated with amoxicillin and $31 \%(4 / 13,95 \%$ CI $12.7 \%-57.6 \%$; p $=0.19$ ) of those treated with ampicillin (Table 3). CS cases were frequently found among non-Japanese pregnant women $(60 \%$ [3/5], 95\% CI $23.1 \%-88.2 \%)$.

In the subgroup of women initiating syphilis treatment $\geq 60$ days before delivery, $14 \%$ (8/56 95\% CI $7.4 \%-25.7 \%$ ) had pregnancies resulting in CS cases (Table 3). In the subgroup initiating treatment $<60$ days before delivery, $43 \%$ (6/14, 95\% CI 21.4\%-67.4\%) had pregnancies resulting in CS. The proportions of women with early and late syphilis were not different between these 2 treatment initiation subgroups ( $p=$ 0.57 by $x^{2}$ test). Among those who initiated syphilis treatment $\geq 60$ days before delivery, $11 \%$ (5/45, 95\% CI $4.8 \%-23.5 \%)$ of those treated with amoxicillin and $27 \%(3 / 11,95 \%$ CI $9.7 \%-56.6 \% ; p=0.11)$ of those treated with ampicillin had pregnancies resulting in CS cases.

Because no CS cases developed among pregnant women with early syphilis, we applied exact logistic regression (27) to estimate exact odds ratios (ORs) and $95 \%$ CIs associated with various factors. Analyses showed late syphilis was associated with CS cases (late vs. early syphilis, adjusted exact OR 13.5, 95\% CI 2.56- $\infty ; p=0.0025$ ) and that starting treatment $\geq 60$ days before delivery had a protective effect ( $\geq 60$ days vs. $<60$ days, adjusted exact OR $0.11,95 \%$ CI $0-0.69$; p $=0.023$ ) (Appendix Table 2).

Discontinuation of antimicrobial drug treatment occurred because of adverse events in $3(3.8 \%)$ of 80 pregnant women. All 3 started amoxicillin and switched to other antimicrobial drugs because of skin rash only, itching only, or dizziness only; 1 of 3 of these patients had a newborn with a CS diagnosis. 
Table 1. Characteristics of pregnant women with active syphilis treated with amoxicillin or ampicillin, by CS birth outcome, Japan, 2010-2018*

\begin{tabular}{|c|c|c|c|c|}
\hline Characteristic & All cases, $n=80$ & CS cases, $\uparrow n=15$ & $\begin{array}{c}\text { Cases of live birth } \\
\text { without CS, } n=56 \ddagger\end{array}$ & $p$ value $\S$ \\
\hline Age, $y$ & $23(21-27)$ & $24(22-26)$ & $26(22-29.5)$ & 0.46 \\
\hline Japanese & $75 / 80(94)$ & $12 / 15(80)$ & $54 / 56(96)$ & 0.06 \\
\hline HIV co-infection & $1 / 78(1)$ & $0 / 15$ & $1 / 56(2)$ & 1.00 \\
\hline \multicolumn{5}{|l|}{ Syphilis stage } \\
\hline Early syphilis & $31 / 80(39)$ & $0 / 15$ & $26 / 56(46)$ & \\
\hline Primary & $5 / 80(6)$ & $0 / 15$ & $5 / 56(9)$ & \\
\hline Secondary & $19 / 80(24)$ & $0 / 15$ & $14 / 56(25)$ & \\
\hline Early latent & $7 / 80(9)$ & $0 / 15$ & $7 / 56(13)$ & \\
\hline Late syphilis & $49 / 80(61)$ & $15 / 15(100)$ & $30 / 56(54)$ & \\
\hline Late latent & $4 / 80(5)$ & $0 / 15$ & $4 / 56(7)$ & \\
\hline Latent with unknown duration & $45 / 80(56)$ & $15 / 15(100)$ & $26 / 56(46)$ & \\
\hline \multicolumn{5}{|l|}{ Diagnosis and treatment } \\
\hline Rapid plasma reagin titer at diagnosis & $51(29-72)$ & $58(28-105)$ & $51(27.1-71.8)$ & 0.59 \\
\hline Amoxicillin & $66 / 80(82.5)$ & $11 / 15(73)$ & $47 / 56(84)$ & 0.45 \\
\hline Ampicillin & $14 / 80(17.5)$ & $4 / 15(27)$ & $9 / 56(16)$ & \\
\hline Antimicrobial drug dosage, $\mathrm{mg} / \mathrm{d}$ & $1,500(1,500-1,500)$ & $1,500(1,500-1,500)$ & $1,500(1,500-1,500)$ & 0.88 \\
\hline Received $1,500 \mathrm{mg} / \mathrm{d}$ & $61 / 80(76)$ & $13 / 15(87)$ & $39 / 56(70)$ & 0.20 \\
\hline Co-administered probenecid & $3 / 75(4)$ & $1 / 15(7)$ & $2 / 56(4)$ & 0.51 \\
\hline Total duration of treatment, $\mathrm{d} \boldsymbol{T}$ & $60(29-90)$ & $70(37-101)$ & $56(28-90)$ & 0.49 \\
\hline Duration of treatment at delivery, $\mathrm{d}$ ा & $56(28-86)$ & $68(4-100)$ & $56(28-84)$ & 0.84 \\
\hline Gestational age at diagnosis, wk & $13.8(11.7-26.3)$ & $18.1(12.3-34)$ & $13.2(11.5-21.6)$ & 0.082 \\
\hline Gestational age at treatment, wk I & $15.8(13.0-27.1)$ & $18.3(13.6-34.3)$ & $15.9(13.4-25.4)$ & 0.22 \\
\hline Gestational age at delivery, wk & $39.1(37.9-40.6)$ & $38.6(35.6-40.9)$ & $39.1(37.9-40.3)$ & 0.38 \\
\hline Time from treatment to delivery, wkT & $21.9(8.5-26.3)$ & $15.7(0.4-25.9)$ & $23(16.7-27.2)$ & 0.026 \\
\hline Started treatment $<60 \mathrm{~d}$ before delivery $\mathbb{T}$ & $14 / 69(20)$ & $6 / 14(43)$ & $8 / 56(14)$ & 0.027 \\
\hline \multicolumn{5}{|l|}{ Birth outcomes } \\
\hline Birth weight, gा & $2,936(2,580-3,156)$ & $2,704(1,797-3,085)$ & $2,959(2,641-3,180)$ & 0.094 \\
\hline Low birth weight, $<2,500 \mathrm{~g}$ & $11 / 69(16)$ & $4 / 13(31)$ & $7 / 56(13)$ & 0.20 \\
\hline Very low birth weight, $<1,500 \mathrm{~g}$ & $3 / 69(4)$ & $3 / 13(23)$ & $0 / 56$ & 0.005 \\
\hline \multicolumn{5}{|c|}{$\begin{array}{l}\text { "Values are no./total (\%) or median (interquartile range). Pregnancy outcome was missing for } 9 \text { patients. CS, congenital syphilis. } \\
\text { tCS cases include newborns with CS diagnoses, miscarriages, and stillbirths. } \\
\text { fOne pregnant woman delivered twins. The twin delivery was acknowledged as } 1 \text { birth outcome, and the birth weight of the first child born was used for } \\
\text { the table and analyses. } \\
\text { §Student } t \text {-test for continuous variables and either } \chi^{2} \text { test or Fisher exact test for categorical variables. } \\
\text { IDenominators varied because of missing data. Anywhere from } 1 \text { to } 11 \text { cases might be missing. }\end{array}$} \\
\hline
\end{tabular}

All 15 women who had CS pregnancy outcomes had latent syphilis of unknown duration (Appendix Table 1). The 13 newborns with CS diagnoses had the following findings: FTA-ABS test results positive for serum $\operatorname{IgM}(\mathrm{n}=10)$, positive serum RPR titers $(\mathrm{n}=$ $5)$, CSF findings $(\mathrm{n}=3)$, very low $(<1,500 \mathrm{~g} ; \mathrm{n}=2)$ or extremely low $(<1,000 \mathrm{~g} ; \mathrm{n}=1)$ birth weight, clinical signs compatible with CS $(\mathrm{n}=2)$, and cardiac anomaly $(\mathrm{n}=1)$. (Appendix Table 1$)$.

A 4-fold decrement in serum RPR titer was achieved in $35(49 \%)$ pregnant women by delivery, $41(53 \%)$ at 6 months after treatment, and $46(82 \%)$ at 1 year after treatment (Table 4). The percentage of CS cases was not significantly different between pregnant women with $(16 \%, \mathrm{n}=5)$ and without $(23 \%, \mathrm{n}=8)$ a 4 -fold titer decline at delivery $(p=0.54)$. However, RPR titers declined $\geq 4$-fold in $91 \%(21 / 23)$ of women with early syphilis (group with 0 CS cases) and 76\% (25/33) of women with late syphilis (group with 15 CS cases) (Table 4). The percentage who achieved a 4-fold decrement was higher among those with early syphilis than among those with late syphilis for all 3 endpoints (by delivery, 58\% early vs. $44 \%$ late; 6 months, $67 \%$ early vs. $45 \%$ late; 1 year, $91 \%$ early vs. $76 \%$ late). Among those who started treatment $\geq 60$ days before delivery, $63 \%(35 / 56)$ had late syphilis, and $81 \%(35 / 43)$ achieved a 4 -fold RPR titer decline by 1 year after treatment (Tables 3,4$)$.

\section{Discussion}

In this nationwide, multicenter retrospective study, we evaluated effectiveness and tolerability of oral amoxicillin or ampicillin for treatment of pregnant women with active syphilis and prevention of MTCT of syphilis. Overall, 21\% (15/71) of pregnancies resulted in CS cases, which we defined to include CS diagnoses in live newborns, miscarriages, and stillbirths. Among pregnancies in women who initiated syphilis treatment $\geq 60$ days before delivery, $14 \%(8 / 56)$ resulted in CS cases. However, no CS cases were observed among the subgroup with early syphilis $(\mathrm{n}=26)$, suggesting that oral amoxicillin with 1,500-mg dosing (500 $\mathrm{mg} 3 \times / \mathrm{d})$ for 30 days could be an optional regimen for treatment of pregnant women with early syphilis when intramuscular BPG is not available. In this study, we found a 
high percentage of CS cases (33\%, 15/45) among those with late syphilis treated with oral amoxicillin or ampicillin, suggesting these regimens should not be used in this population. Late initiation of treatment was also associated with CS independent of syphilis stage. Although further studies evaluating drug adherence are warranted before any recommendation can be made, these findings are valuable for implicating some alternative treatments, considering reports of syphilis increases among heterosexual populations, reports of CS increases in various countries (including Japan and the United States), and national shortages of BPG $(28,29)$.

Our study has 3 strengths. First, we evaluated effectiveness of oral amoxicillin or ampicillin for the treatment of pregnant women with active syphilis and prevention of MTCT. The finding that $1,500 \mathrm{mg}$ of oral amoxicillin for 30 days might be effective in treating pregnant women with early syphilis and preventing CS is clinically useful because intramuscular BPG, the only recommended regimen for this population, is not always available because of shortages or stockouts $(3,9)$. High-dose oral amoxicillin $(3,000 \mathrm{mg} / \mathrm{d})$ plus probenecid has been reported to effectively treat syphilis in nonpregnant patients with HIV infection with a serologic effectiveness of $97.5 \%$ for early syphilis and $90.8 \%$ for late syphilis (10). Further studies are warranted to determine efficacy, optimal dosing, duration, and the need for co-administration of probenecid with oral amoxicillin as an alternative regimen for both early and late syphilis in pregnant women.
Second, the well-established and strict inclusion criteria of having both serum RPR titers $\geq 8$ and positive treponemal test results, regardless of symptoms, for active syphilis was a strength of this study $(10,15,19)$. Application of these criteria enabled us to exclude those with low RPR titers who might have had previous antimicrobial drug treatment or those with serofast status, for whom further treatment is not required (30). This inclusion criterion was necessary to exclude persons who had past (not current) infections, considering that various antimicrobial drugs can treat syphilis $(30,31)$ and that patients can receive effective treatment for syphilis without recognizing it.

Third, we evaluated not only MTCT of syphilis but also treatment effectiveness of oral amoxicillin or ampicillin in women using serology. Serologic effectiveness 1 year after treatment was $82 \%$ overall and $91 \%$ among early syphilis cases, suggesting greater effectiveness of this regimen for early syphilis. Furthermore, as was the case for the report by Rac et al. (25), we showed that the percentage of CS cases was not different between patients with and without a 4 -fold titer decline at delivery.

The effectiveness of antimicrobial drugs for the prevention of MTCT of syphilis needs to be carefully considered because evidence is limited and diagnostic criteria for pregnant women with syphilis and CS vary across studies $(5,8,25)$. We included time between treatment and delivery and syphilis staging in analyses

\begin{tabular}{|c|c|c|c|}
\hline Category & Early syphilis, $n=31$ & Late syphilis, $n=49$ & $\mathrm{p}$ value† \\
\hline Age, y & $26(21-28)$ & $25(22-27)$ & 0.63 \\
\hline Japanese & 29/31 (94) & $46 / 49(94)$ & 1.00 \\
\hline HIV co-infection & $0 / 31$ & $1 / 49(2)$ & 1.00 \\
\hline \multicolumn{4}{|l|}{ Diagnosis and treatment } \\
\hline Rapid plasma reagin titer at diagnosis & $44(27-64)$ & $58(29-83)$ & 0.64 \\
\hline Amoxicillin & 28/31 (90) & $38 / 49(78)$ & 0.23 \\
\hline Ampicillin & $3 / 31(10)^{\prime}$ & $11 / 49(22)$ & \\
\hline Antimicrobial drug dosage, $\mathrm{mg} / \mathrm{d}$ & $1,500(1,500-1,500)$ & $1,500(1,500-1,500)$ & 0.56 \\
\hline Received $1,500 \mathrm{mg} / \mathrm{d}$ & 26/31 (84) & $35 / 49(71)$ & 0.22 \\
\hline Total duration of treatment, $\mathrm{d} \ddagger$ & $30(28-64)$ & $78(51-104)$ & 0.004 \\
\hline Duration of treatment at delivery, $\mathrm{d} \ddagger$ & $30(26-56)$ & $70(29-98)$ & 0.016 \\
\hline Gestational age at diagnosis, wk $\ddagger$ & $15.1(11.1-27.5)$ & $13.3(12-25.6)$ & 0.70 \\
\hline Gestational age at treatment, wk $\ddagger$ & $17.3(13.3-28.7)$ & $14.7(13-26)$ & 0.62 \\
\hline Gestational age at delivery, wk $\ddagger$ & $39.4(38.1-40.6)$ & $38.9(37.7-40.5)$ & 0.10 \\
\hline Time from treatment to delivery, wk $\ddagger$ & $15.7(0.4-25.9)$ & $23(16.7-27.2)$ & 0.73 \\
\hline \multicolumn{4}{|l|}{ Birth outcomes } \\
\hline Live birth with congenital syphilis diagnosis & $0 / 26$ & $13 / 45(29)$ & 0.009 \\
\hline Stillbirth & $0 / 26$ & $1 / 45(2)$ & \\
\hline Miscarriage & $0 / 26$ & $1 / 45(2)$ & \\
\hline Birth weight, gł & $2,902(2,652-3,184)$ & $2,942(2,555-3,155)$ & 0.88 \\
\hline Low birth weight, $<2,500 \mathrm{~g} \ddagger$ & $3 / 26(12)$ & $8 / 45(18)$ & 0.52 \\
\hline Very low birth weight, $<1,500 \mathrm{~g} \ddagger$ & $0 / 26$ & $3 / 45(7)$ & 0.29 \\
\hline
\end{tabular}

*Values are no./total (\%) or median (interquartile range).

†Used the Student $t$-test for continuous variables and either the $\chi^{2}$ test or Fisher exact test for categorical variables.

fDenominators varied because of missing data. Anywhere from 1 to 11 cases might be missing. 
Table 3. Birth outcomes of pregnant women with active syphilis treated with oral amoxicillin or ampicillin, Japan, 2010-2018*

\begin{tabular}{|c|c|c|c|c|c|c|c|c|}
\hline \multirow[b]{2}{*}{ Category } & \multirow[b]{2}{*}{$\begin{array}{c}\text { Total } \\
\text { no. }\end{array}$} & \multirow[b]{2}{*}{$\begin{array}{l}\text { No. } \\
\text { missing }\end{array}$} & \multirow{2}{*}{$\begin{array}{l}\text { No. live births } \\
\text { without CS } \\
\text { diagnosis }\end{array}$} & \multicolumn{3}{|c|}{ No. adverse outcomes } & \multirow{2}{*}{$\begin{array}{c}\text { Adverse } \\
\text { outcomes, \% } \\
(95 \% \mathrm{Cl})\end{array}$} & \multirow[b]{2}{*}{$\mathrm{p}$ value } \\
\hline & & & & $\begin{array}{l}\text { Live births with } \\
\text { CS diagnosis }\end{array}$ & Miscarriages & Stillbirths & & \\
\hline All patients & 80 & 9 & 56 & 13 & 1 & 1 & $21.1(13.2-32)$ & \\
\hline Early syphilis & 31 & 5 & 26 & 0 & 0 & 0 & $0(0-12.9)$ & $<0.001$ \\
\hline Late syphilis & 49 & 4 & 30 & 13 & 1 & 1 & $33.3(21.4-47.9)$ & \\
\hline Amoxicillin & 66 & 8 & 47 & 9 & 1 & 1 & $19.0(10.9-30.9)$ & 0.19 \\
\hline Ampicillin & 14 & 1 & 9 & 4 & 0 & 0 & $30.8(12.7-57.6)$ & \\
\hline Japanese & 75 & 9 & 54 & 10 & 1 & 1 & $18.2(10.7-29.1)$ & 0.033 \\
\hline Non-Japanese & 5 & 0 & 2 & 3 & 0 & 0 & $60.0(23.1-88.2)$ & \\
\hline \multicolumn{9}{|c|}{ Patients starting syphilis treatment $>60$ days before delivery $\ddagger$} \\
\hline All & 57 & 1 & 48 & 8 & 0 & 0 & $14.2(7.4-25.7)$ & \\
\hline Early syphilis & 21 & 0 & 21 & 0 & 0 & 0 & $0(0-15.5)$ & 0.017 \\
\hline Late syphilis & 36 & 1 & 27 & 8 & 0 & 0 & $22.9(12.1-39)$ & \\
\hline Amoxicillin & 46 & 1 & 40 & 5 & 0 & 0 & $11.1(4.8-23.5)$ & 0.11 \\
\hline Ampicillin & 11 & 0 & 8 & 3 & 0 & 0 & $27.3(9.7-56.6)$ & \\
\hline Japanese & 54 & 1 & 47 & 6 & 0 & 0 & $11.3(5.3-22.6)$ & 0.026 \\
\hline Non-Japanese & 3 & 0 & 1 & 2 & 0 & 0 & $66.7(20.8-93.9)$ & \\
\hline \multicolumn{9}{|c|}{ Patients starting syphilis treatment $<60$ days before delivery } \\
\hline All & 14 & 0 & 8 & 5 & 0 & 1 & $42.9(21.4-67.4)$ & \\
\hline Early syphilis & 5 & 0 & 5 & 0 & 0 & 0 & $0(0-43.5)$ & 0.028 \\
\hline Late syphilis & 9 & 0 & 3 & 5 & 0 & 1 & $66.7(35.4-87.9)$ & \\
\hline Amoxicillin & 13 & 0 & 8 & 4 & 0 & 1 & $38.5(17.7-64.5)$ & 0.43 \\
\hline Ampicillin & 1 & 0 & 0 & 1 & 0 & 0 & $100(20.7-100)$ & \\
\hline Japanese & 12 & 0 & 7 & 4 & 0 & 1 & $41.7(19.3-68.1)$ & 0.86 \\
\hline Non-Japanese & 2 & 0 & 1 & 1 & 0 & 0 & $50.0(9.5-90.6)$ & \\
\hline \multicolumn{9}{|c|}{$\begin{array}{l}\text { *Nine cases (including } 3 \text { cases of induced abortion) were excluded from this analysis because data on birth outcomes were not available. One } \\
\text { miscarriage case is missing in the subgroup analysis because the exact date of miscarriage was unknown, CS, congenital syphilis. } \\
\text { †Used either the } \chi^{2} \text { test or Fisher exact test to measure the association between cases of live birth without CS diagnosis and cases of live birth with CS } \\
\text { diagnosis, miscarriage, and stillbirth. } \\
\text { tOutcome data for } 1 \text { patient is missing. Outcome data (whether CS or not) was not known because this patient was referred to another hospital before } \\
\text { delivery. Because the birth date of this patient's infant was reported and available, we could group this patient with the appropriate group, which was the } \\
\text { group that started syphilis treatment } \geq 60 \text { days before delivery. }\end{array}$} \\
\hline
\end{tabular}

because these parameters influence MTCT; transmission is more likely to occur during early syphilis stages and among women with late treatment $(30,32)$. The CDC-recommended regimen of intramuscular BPG once for early syphilis and three times at 1-week intervals for late syphilis was reported to prevent $98.2 \%$ of CS cases $(97.1 \%$ for early syphilis, $100 \%$ for late syphilis including latent syphilis of unknown duration) among a cohort of pregnant women with syphilis diagnosed during 1987-1989 (5). However, note that the authors of that investigation applied loose inclusion criteria (positive for treponemal antibody and positive by either the venereal disease research laboratory test or the RPR test); their diagnostic criteria for CS was strict (5). That group of authors subsequently reported that, among a cohort of pregnant women with syphilis treated during 1981-2011 by CDC guidelines, $18 \%$ of infants subsequently required treatment for CS (25). Only 1 case series has been published on the use of ceftriaxone to treat pregnant women with syphilis and to prevent CS (8). In that study, 2 courses of intramuscular ceftriaxone with 250-mg dosing for 7-10 days was evaluated in 11 pregnant women with early syphilis, and this treatment resulted in no CS diagnoses.

Our study demonstrates the need for comparative trials to evaluate the use of amoxicillin in the preven- tion of MTCT of syphilis before recommending this regimen as an alternative to BPG for pregnant women with syphilis. Considering our study results, healthcare authorities in Japan and other countries where intramuscular BPG is not recommended should consider making intramuscular BPG available. When intramuscular BPG is not available to treat pregnant women with syphilis, physicians need to make difficult decisions regarding alternative regimens, often following recommendations for neurosyphilis, such as intravenous aqueous crystalline penicillin $G$ or intravenous or intramuscular ceftriaxone, treatments with limited clinical evidence available on efficacy.

We found that $17 \%$ of pregnant women were treated with oral ampicillin, even though oral amoxicillin is favored over oral ampicillin for most indications because of greater bioavailability $(31,33)$. Oral ampicillin is also used to prevent group B Streptococcus at some facilities in Japan, despite guidelines recommending the injectable form of ampicillin for such indications $(11,34)$. Our study results showed that the effectiveness of oral amoxicillin and ampicillin were not significantly different; however, our sample size was small.

Our study has several limitations. First, the retrospective nature of the study could have introduced 
Table 4. Serologic outcomes of pregnant women with active syphilis treated with amoxicillin or ampicillin, Japan, 2010-2018, by delivery, 6 months after treatment, and 1 year after treatment*

\begin{tabular}{|c|c|c|c|}
\hline \multirow[b]{2}{*}{ Patient group } & \multicolumn{3}{|c|}{ No./total (\%) with $>4$-fold decrement in RPR titer } \\
\hline & By delivery & 6 months after treatment & 1 year after treatment \\
\hline All & $35 / 71(49)$ & $41 / 77(53)$ & $46 / 56(82)$ \\
\hline Live birth without CS diagnosis & $27 / 54(50)$ & $29 / 54(54)$ & $31 / 39(80)$ \\
\hline CS cases $\dagger$ & $5 / 13(39)$ & $6 / 15(40)$ & $9 / 11(82)$ \\
\hline Early syphilis & $15 / 26(58)$ & $20 / 30(67)$ & $21 / 23(91)$ \\
\hline Late syphilis & $20 / 45(44)$ & $21 / 47(45)$ & $25 / 33(76)$ \\
\hline Amoxicillin & $27 / 58(47)$ & $32 / 63(51)$ & $36 / 44(82)$ \\
\hline Ampicillin & $8 / 13(62)$ & $9 / 14(64)$ & 10/12 (83) \\
\hline Japanese & $34 / 66(52)$ & $39 / 72(54)$ & $44 / 52(85)$ \\
\hline Non-Japanese & $1 / 5(20)$ & $2 / 5(40)$ & $2 / 4(50)$ \\
\hline Started syphilis treatment $\geq 60$ days before delivery & $32 / 55(58)$ & $32 / 55(58)$ & $35 / 43(81)$ \\
\hline Started syphilis treatment $<60$ days before delivery & 0 & $5 / 17(29)$ & $6 / 8(75)$ \\
\hline
\end{tabular}

some selection and information bias, and variables were missing for some mothers and infants. Second, data on adherence of patients to the prescribed regimens were not available, could not be evaluated, and could have affected our findings. Third, the definition of CS is not universal; diagnostic criteria can differ in national surveillance guidelines of other countries, limiting the comparativeness of our findings with other studies $(4,12,22)$. In this study, we followed the reporting criteria set by the Japanese Ministry of Health, Labour, and Welfare, which includes the criterion of FTA-ABS test positivity for serum IgM in newborns $(12,20)$. In contrast to IgG, IgM does not cross the placenta, making IgM in the serum of newborns a reliable marker with high specificity for CS diagnosis $(21,35)$. However, a sufficiently sensitive and specific IgM assay is not commercially available (4). CDC guidelines do not include FTA-ABS test positivity for serum IgM in newborns as a criterion for CS diagnosis (4), whereas the Japan and the European Centre for Disease Prevention and Control guidelines do $(12,20,22)$. In our study, misclassification of infants with CS could have been possible because evaluation (including laboratory testing) of newborns was not always rigorously performed. CS can cause long bone and CSF findings; these criteria are used in the United States for CS diagnosis (4). Because long bone radiographs are not included in the reporting criteria for CS in Japan, these procedures are infrequently performed, and results were not available for review in this analysis. Among 71 cases with available birth outcomes, 60 had serum RPR test results and only 6 had CSF results (3 with remarkable findings). Fourth, even though we classified latent syphilis of unknown duration as late syphilis, this category could have included early latent syphilis; clearly distinguishing between these 2 groups is difficult. Last, our results should be interpreted with caution because of the small sample size.
In conclusion, we evaluated the effectiveness and tolerability of oral amoxicillin and ampicillin for the treatment of pregnant women with active syphilis and prevention of MTCT of syphilis in Japan. Although we cannot recommend oral amoxicillin or ampicillin as alternative regimens for the treatment of pregnant women with syphilis, this analysis suggests that $1,500 \mathrm{mg} /$ day of oral amoxicillin for 30 days could effectively treat early syphilis and prevent CS. Further studies, preferably controlled comparative trials, are needed to establish the efficacy of oral amoxicillin as an alternative regimen for the treatment of pregnant women with syphilis and the prevention of CS.

\section{Acknowledgements}

The authors thank the following gynecologists and obstetricians at 44 hospitals across Japan who kindly collected the data and fulfilled the case report forms for this study: Yukio Wakui, Yoshihiro Akimoto, Yusaku Kumagai, Yuki Shimoda, Yutaka Fujiki, Maiko Ichikawa, Takashi Watanabe, Hidenori Sasa, Tomoko Amagata, Hisayo Fujimura, Yoshinori Iitsuka, Hironobu Hyodo, Miki Goto, Hideyuki Oda, Noriko Sato, Yasuko Nagasaka, Masahiro Shiba, Hiroko Tsuchiya, Junko Mochizuki, Soichiro Obata, Toru Arase, Hiroki Waki, Yasuko Nemoto, Tomoaki Kano, Saki Inuzuka, Noriko Kato, Kazuhiro Higuchi, Yasuyuki Noguchi, Shima Takamura, Masako Sawada, Masahiro Ohashi, Kuniko Hanabusa, Ikuko Sawada, Kayoko Harada, Masako Tomimoto, Yoshinori Takeda, Taihei Tsunemi, Yoshimasa Onohara, Hiroko Kurioka, Mika Sugihara, Yuriko Omori, Masakatsu Sase, Masaki Goto, Naotoshi Honda, Shoichi Kawakami, Junya Miyoshi, and Tadakazu Uesato. The authors would also like to thank Makoto Ohnishi, Motoyuki Tsuboi, Yosuke Inaba, and Naoko Tomita for their invaluable support for this study.

This work was supported by the Ministry of Health, Labour, and Welfare of Japan. 


\section{About the Author}

Dr. Nishijima is a clinician-researcher at the National Center for Global Health and Medicine, Tokyo, Japan. His research interests include clinical trials and observational studies on HIV and sexually transmitted infections.

\section{References}

1. Korenromp EL, Rowley J, Alonso M, Mello MB, Wijesooriya NS, Mahiané SG, et al. Global burden of maternal and congenital syphilis and associated adverse birth outcomes-estimates for 2016 and progress since 2012 [Erratum in: PLoS One. 2019;14:e0219613]. PLoS One. 2019;14:e0211720. http://dx.doi.org/10.1371/journal. pone. 0211720

2. Lawn JE, Blencowe H, Waiswa P, Amouzou A, Mathers C, Hogan D, et al.; Lancet Ending Preventable Stillbirths Series study group; Lancet Stillbirth Epidemiology investigator group. Stillbirths: rates, risk factors, and acceleration towards 2030. Lancet. 2016;387:587-603. http:/ / dx.doi.org/10.1016/ S0140-6736(15)00837-5

3. World Health Organization. WHO guidelines for the treatment of Treponema pallidum (syphilis). 2016 [cited 2020 Jan 30]. http:/ / apps.who.int/iris/bitstream/10665/ 249572/1/9789241549806-eng.pdf

4. Workowski KA, Bolan GA; Centers for Disease Control and Prevention. Sexually transmitted diseases treatment guidelines, 2015. MMWR Recomm Rep. 2015;64(RR-03):1-137.

5. Alexander JM, Sheffield JS, Sanchez PJ, Mayfield J, Wendel GD Jr. Efficacy of treatment for syphilis in pregnancy. Obstet Gynecol. 1999;93:5-8.

6. Roberts CP, Raich A, Stafylis C, Klausner JD. Alternative treatments for syphilis during pregnancy. Sex Transm Dis. 2019;46:637-40. http:/ / dx.doi.org/10.1097/ OLQ.0000000000001050

7. Molini BJ, Tantalo LC, Sahi SK, Rodriguez VI, Brandt SL, Fernandez MC, et al. Macrolide resistance in Treponema pallidum correlates with $23 \mathrm{~S}$ rDNA mutations in recently isolated clinical strains. Sex Transm Dis. 2016;43:579-83. http:/ /dx.doi.org/10.1097/OLQ.0000000000000486

8. Zhou P, Gu Z, Xu J, Wang X, Liao K. A study evaluating ceftriaxone as a treatment agent for primary and secondary syphilis in pregnancy. Sex Transm Dis. 2005;32:495-8. http://dx.doi.org/10.1097/01.olq.0000170443.70739.cd

9. Nurse-Findlay S, Taylor MM, Savage M, Mello MB, Saliyou S, Lavayen M, et al. Shortages of benzathine penicillin for prevention of mother-to-child transmission of syphilis: an evaluation from multi-country surveys and stakeholder interviews. PLoS Med. 2017;14:e1002473. http://dx.doi.org/10.1371/journal.pmed.1002473

10. Tanizaki R, Nishijima T, Aoki T, Teruya K, Kikuchi Y, Oka S, et al. High-dose oral amoxicillin plus probenecid is highly effective for syphilis in patients with HIV infection. Clin Infect Dis. 2015;61:177-83. http://dx.doi.org/10.1093/cid/civ270

11. Japan Society of Obstetrics and Gynecology; Japan Association of Obstetricians and Gynecologists. The Japanese obstetrics and gynecology care guidelines [in Japanese]. 2017 [cited 2020 Jan 30]. http:/ / www.jsog.or.jp/activity/pdf/ gl_fujinka_2017.pdf

12. Japanese Society for Sexually Transmitted Infections. The Japanese STI diagnosis and treatment guidelines [in Japanese]. 2016 [cited 2020 Jan 30]. http:/ /jssti.umin.jp/pdf/ guideline-2016_v2.pdf

13. Katanami Y, Hashimoto T, Takaya S, Yamamoto K, Kutsuna S, Takeshita N, et al. Amoxicillin and ceftriaxone as treatment alternatives to penicillin for maternal syphilis. Emerg Infect Dis. 2017;23:827-9. http:/ / dx.doi.org/10.3201/ eid2305.161936

14. Takamatsu K, Kitawaki J. Annual report of the Women's Health Care Committee, Japan Society of Obstetrics and Gynecology, 2017. J Obstet Gynaecol Res. 2018;44:13-26. http://dx.doi.org/10.1111/jog.13470

15. Kenyon C, Lynen L, Florence E, Caluwaerts S, Vandenbruaene M, Apers L, et al. Syphilis reinfections pose problems for syphilis diagnosis in Antwerp, Belgium - 1992 to 2012. Euro Surveill. 2014;19:20958. http:/ / dx.doi.org/ 10.2807/1560-7917.ES2014.19.45.20958

16. Marra CM, Maxwell CL, Smith SL, Lukehart SA, Rompalo AM, Eaton M, et al. Cerebrospinal fluid abnormalities in patients with syphilis: association with clinical and laboratory features. J Infect Dis. 2004;189:369-76. http:/ / dx.doi.org/10.1086/381227

17. Zetola NM, Engelman J, Jensen TP, Klausner JD. Syphilis in the United States: an update for clinicians with an emphasis on HIV coinfection. Mayo Clin Proc. 2007;82: 1091-102. http://dx.doi.org/10.4065/82.9.1091

18. Lee JH, Lim CS, Lee MG, Kim HS. Comparison of an automated rapid plasma reagin (RPR) test with the conventional RPR card test in syphilis testing. BMJ Open. 2014;4:e005664. http://dx.doi.org/10.1136/ bmjopen-2014-005664

19. Tsuboi M, Nishijima T, Aoki T, Teruya K, Kikuchi Y, Gatanaga H, et al. Usefulness of automated latex turbidimetric rapid plasma reagin test for diagnosis and evaluation of treatment response in syphilis in comparison with manual card test: a prospective cohort study. J Clin Microbiol. 2018;56:e01003-18. http://dx.doi.org/10.1128/ JCM.01003-18

20. Government of Japan. Case report form of congenital syphilis [in Japanese] [cited 2020 Jan 30]. https:/ / www. mhlw.go.jp/bunya/kenkou/kekkaku-kansenshou11/ pdf/01-05-11-3-b.pdf

21. Herremans T, Kortbeek L, Notermans DW. A review of diagnostic tests for congenital syphilis in newborns. Eur J Clin Microbiol Infect Dis. 2010;29:495-501. http:/ /dx.doi.org/10.1007/s10096-010-0900-8

22. European Union. Decisions. Commission implementing decision (EU) 2018/945 of 22 June 2018 on the communicable diseases and related special health issues to be covered by epidemiological surveillance as well as relevant case definitions. Off J Eur Union. 2018;61:L170/44-5 [cited 2020 Jan 30] https:/ / eur-lex.europa.eu/legal-content/EN/TXT/ PDF/?uri=OJ:L:2018:170:FULL\&from

23. Cheng JQ, Zhou H, Hong FC, Zhang D, Zhang YJ, Pan P, et al. Syphilis screening and intervention in 500,000 pregnant women in Shenzhen, the People's Republic of China. Sex Transm Infect. 2007;83:347-50. http:// dx.doi.org/10.1136/ sti.2006.023655

24. Janier M, Hegyi V, Dupin N, Unemo M, Tiplica GS, Potočnik M, et al. 2014 European guideline on the management of syphilis. J Eur Acad Dermatol Venereol. 2014;28:1581-93. http://dx.doi.org/10.1111/jdv.12734

25. Rac MW, Bryant SN, Cantey JB, McIntire DD, Wendel GD Jr, Sheffield JS. Maternal titers after adequate syphilotherapy during pregnancy. Clin Infect Dis. 2015;60:686-90. http://dx.doi.org/10.1093/cid/ciu920

26. World Health Organization. Global guidance on criteria and processes for validation: elimination of mother-to-child transmission of HIV and syphilis. 2nd edition. 2017 [cited 2020 Jan 30]. https:/ / www.who.int/reproductivehealth/ publications/emtct-hiv-syphilis 
27. Mehta CR, Patel NR. Exact logistic regression: theory and examples. Stat Med. 1995;14:2143-60. http:/ / dx.doi.org/ 10.1002/sim.4780141908

28. Takahashi T, Arima Y, Yamagishi T, Nishiki S, Kanai M, Ishikane $M$, et al. Rapid increase in reports of syphilis associated with men who have sex with women and women who have sex with men, Japan, 2012 to 2016. Sex Transm Dis. 2018;45:139-43. http://dx.doi.org/10.1097/ OLQ.0000000000000768

29. Centers for Disease Control and Prevention, National Center for HIV/AIDS, Viral Hepatitis, STD, and TB Prevention. Sexually transmitted disease surveillance 2017. 2018 Sep [cited 2019 Jun 10]. https:/ / www.cdc.gov/std/stats17/ 2017-STD-Surveillance-Report_CDC-clearance-9.10.18.pdf

30. Clement ME, Okeke NL, Hicks CB. Treatment of syphilis: a systematic review. JAMA. 2014;312:1905-17. http:/ / dx.doi.org/10.1001/jama.2014.13259

31. Bennett J, Dolin R, Blaser MJ. Mandell, Douglas, and Bennett's principles and practice of infectious diseases. 8th edition. London: Churchill Livingstone Elsevier; 2014

32. Sheffield JS, Sánchez PJ, Morris G, Maberry M, Zeray F, McIntire DD, et al. Congenital syphilis after maternal treatment for syphilis during pregnancy. Am J Obstet Gynecol. 2002;186:569-73. http:/ /dx.doi.org/10.1067/ mob.2002.121541

33. Klein JO, Finland M. The new penicillins. N Engl J Med. 1963;269:1129-34. http:/ / dx.doi.org/10.1056/ NEJM196311212692107

34. AmericanCollege of Obstetricians and Gynecologists Committee on Obstetric Practice. Prevention of group B streptococcal early-onset disease in newborns: ACOG committee opinion summary, number 797 [Erratum in: Obstet Gynecol. 2020;135:978-9]. Obstet Gynecol. 2020; 135:e51-72. http://dx.doi.org/10.1097/ AOG.0000000000003668

35. Johnston NA. Neonatal congenital syphilis. Diagnosis by the absorbed fluorescent treponemal antibody (IgM) test. Br J Vener Dis. 1972;48:464-9.

Address for correspondence: Kei Kawana, Department of Obstetrics and Gynecology, Nihon University School of Medicine, 30-1 Ohyaguchi Kamicho, Itabashi, Tokyo 173-8610, Japan; email: kkawana-tky@umin.org

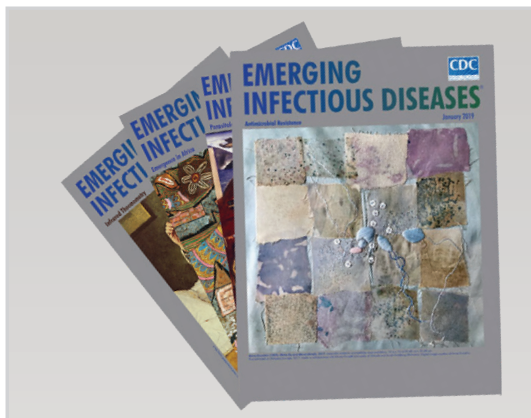

Complexity of the Basic Reproduction Number $\left(R_{0}\right)$

Aeromedical Transfer of Patients with Viral Hemorrhagic Fever

Clinical and Radiologic Characteristics of Human Metapneumovirus Infections in Adults, South Korea

Enterovirus A71 Infection and Neurologic Disease, Madrid, Spain, 2016

Epidemiology of Imported Infectious Diseases, China, 2005-2016

Risk Factors for Elizabethkingia Acquisition and Clinical Characteristics of Patients, South Korea

Effects of Antibiotic Cycling Policy on Incidence of HealthcareAssociated MRSA and Clostridioides difficile Infection in Secondary Healthcare Settings

Association of Increased Receptor-Binding Avidity of Influenza A(H9N2) Viruses with Escape from Antibody-Based Immunity and Enhanced Zoonotic Potential

Variable Protease-Sensitive Prionopathy Transmission to Bank Voles

Zoonotic Source Attribution of Salmonella enterica Serotype Typhimurium Using Genomic Surveillance Data, United States

Multiple Introductions of Domestic Cat Feline Leukemia Virus in Endangered Florida Panthers

Prescription of Antibacterial Drugs for HIV-Exposed, Uninfected Infants, Malawi, 2004-2010

\section{January 2019}

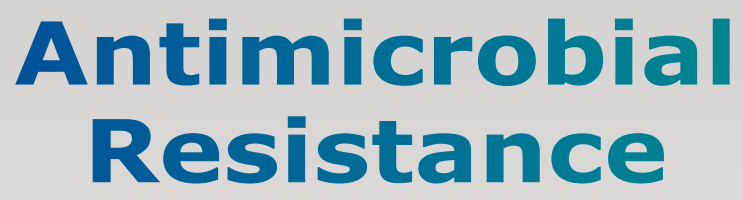

Influenza H5/H7 Virus Vaccination in Poultry and Reduction of Zoonotic Infections, Guangdong Province, China, 2017-18

Higher Viral Load of Emerging Norovirus GII.P16-GII.2 than Pandemic GII.4 and Epidemic GII.17, Hong Kong, China

Autochthonous Transmission of Coccidioides in Animals, Washington, USA

Meat and Fish as Sources of Extended-Spectrum $\beta$-LactamaseProducing Escherichia coli, Cambodia

Oral Transmission of Trypanosoma cruzi, Brazilian Amazon

Avian Influenza A(H9N2) Virus in Poultry Worker, Pakistan, 2015

Puumala Hantavirus Genotypes in Humans, France, 2012-2016

New Multidrug-Resistant Salmonella enterica Serovar Anatum Clone, Taiwan, 2015-2017

Seroepidemiology of Parechovirus A3 Neutralizing Antibodies, Australia, the Netherlands, and United States

Identification of Lonepinella sp. in Koala Bite Wound Infections, Queensland, Australia

Surgical Site Infections Caused by Highly Virulent Methicillin-Resistant Staphylococcus aureus Sequence Type 398, China

Canine Influenza Virus A(H3N2) Clade with Antigenic Variation, China, 2016-2017 